\title{
Effects of testosterone on circadian rhythmicity in old mice
}

\author{
Atsuyoshi Hashimoto ${ }^{1}$. Shingo Fujiki ${ }^{1} \cdot$ Wataru Nakamura ${ }^{2} \cdot$ Takahiro J. Nakamura $^{1}$ (b
}

Received: 27 February 2019 / Accepted: 6 July 2019 / Published online: 12 July 2019

(C) The Physiological Society of Japan and Springer Japan KK, part of Springer Nature 2019

\begin{abstract}
Serum testosterone concentration decreases with age in humans and rodents. Accordingly, old male mice show changes in locomotor activity rhythms: a lengthened free-running period and decreased activity levels among others. To investigate whether testosterone replacement improves the age-related decline in circadian rhythmicity, we examined the effects of testosterone on the circadian rhythms of wheel running activity in old male mice. Intact male C57BL/6J mice (18-22 months old) were subcutaneously implanted with silicone tubes packed with testosterone propionate (TP) or cholesterol. TP treatment significantly decreased the daily wheel running revolutions in a normal light/dark (LD) cycle and in constant darkness (DD), but did not affect the free-running period. The same experiment performed on young male gonadectomized mice (3-5 months old) demonstrated that TP treatment significantly increased activity levels in both LD and DD. These results suggest that testosterone replacement exacerbates the age-related decline in circadian rhythmicity.
\end{abstract}

Keywords Circadian rhythm $\cdot$ Testosterone $\cdot$ Wheel running $\cdot$ Free-running period $\cdot$ PER2::LUC

\section{Introduction}

Serum testosterone concentration decreases with age in both humans and rodents [1-3]. Lower testosterone levels are associated with decreased physical and mental function, increased mortality, and decreased quality of life [4]. Testosterone replacement therapy (TRT) is increasingly being used to alleviate age-related decline in physical functions in elderly men [5]. However, the clinical benefits of TRT in older men are still a subject of debate [6]. Circadian rhythms, like most physiological processes, are profoundly influenced by aging. In humans and other mammals, aging is commonly associated with changes in the quantity and quality of sleep, and with specific sleep disorders, including sleep episode fragmentation [7-9]. In addition, aging decreases the amplitudes of diurnal rhythms in hormone secretion, urine metabolites, and blood pressure in humans

Takahiro J. Nakamura

takahiro@meiji.ac.jp

1 Laboratory of Animal Physiology, School of Agriculture, Meiji University, 1-1-1 Hgashimita, Tama-ku, Kawasaki, Kanagawa 214-8571, Japan

2 Department of Oral-Chrono Physiology, Graduate School of Biomedical Sciences, Nagasaki University, Nagasaki, Nagasaki 852-8588, Japan
[10-12]. In rodents, aging induces changes in locomotor activity rhythms, including decreased amplitude, increased fragmentation, altered free-running periods, slower reentrainment following light-dark cycle shifts, and altered light sensitivity [8, 13-16].

Circadian rhythms are coordinated by a central clock located in the suprachiasmatic nucleus (SCN) of the hypothalamus. The SCN receives environmental light cues from the retinas and adjusts the body to the daily rotation of the earth [17]. SCN lesions abolish circadian rhythms of locomotor activity and hormones in rodents [18], indicating that the SCN plays an important role in physiological and behavioral circadian rhythms. Circadian rhythms are generated within mammalian cells by interlocking transcriptional-translational feedback loops involving a family of clock genes [19]. Period2 (Per2) is a clock gene that shows circadian oscillations in the cells of almost all organs and is used to assess the periods and amplitudes of cellular clocks [20].

Androgen receptors are expressed in the SCN of humans and rodents, rendering the SCN sensitive to androgen treatment [21-25]. With regard to the role of androgens in circadian rhythms, it has been reported that gonadectomy (GDX) lengthens the free-running period of circadian behavioral rhythms and decreases overall activity in young male mice, an effect rescued by testosterone replacement [26]. However, 
this effect was observed only in dim light conditions but not in constant darkness (DD) [27]. To our knowledge, it remains unknown whether androgens directly influence the period and/or amplitude of circadian rhythms of clock gene expression in the SCN.

In this study, we hypothesized that testosterone would improve age-related decline in circadian behavioral rhythms by modulating the period and amplitude of circadian rhythms of clock gene expression in the SCN. To assess our hypothesis, we examined the effects of testosterone propionate (TP) on the circadian rhythms of locomotor activity in old and young mice and the expression rhythms of clock genes in the SCN using ex vivo bioluminescent recordings of PER2::luciferase knock-in (PER2::LUC) mice.

\section{Materials and methods}

\section{Animals and housing}

Male C57BL6/J mice were purchased from Japan SLC (Shizuoka, Japan). Animals were classified into young and old groups and were housed separately until they reached the experimental age. Male heterozygous PER2::LUC mice (C57BL/6J background) were obtained from The Jackson Laboratory (Bar Harbor, ME, USA) and bred for use in bioluminescence recordings. Animals were maintained in a 12:12 h light-dark cycle under controlled air conditions (room temperature, $23 \pm 1{ }^{\circ} \mathrm{C}$; humidity, $50 \pm 10 \%$ ) with food and water available ad libitum. All animal housing and experimental procedures were in accordance with the guidelines of the Japanese Physiological Society and approved by the Institutional Animal Care and Use Committee at the School of Agriculture Meiji University (permission \#: IACUC16-0012).

\section{Surgery: GDX and hormone replacement}

GDX was performed under isoflurane anesthesia (5\% for induction and $1.5-2 \%$ for maintenance). Testes were externalized via laparotomy and then removed after clamping the testicular artery. The incisions were closed with sutures.

Silicone tubes (inner diameter $=2.0 \mathrm{~mm}$, outer diameter $=3.0 \mathrm{~mm}$; Kaneka, Osaka, Japan) were packed with $100 \%$ testosterone propionate (TP; Sigma-Aldrich, St. Louis, MO, USA) or $100 \%$ cholesterol (Sigma-Aldrich; vehicle group), and sealed with silicone adhesive (Konishi, Osaka, Japan). Tubes were $12 \mathrm{~mm}$ long with an additional $3 \mathrm{~mm}$ on each end for the adhesive. This method has been used in animal research for a long time [28, 29], in which TP constantly leaks from the tube [30]. Tubes were washed with $70 \%$ ethanol and primed in $0.9 \%$ sterile saline at $37^{\circ} \mathrm{C}$ overnight and then implanted subcutaneously under isoflurane anesthesia during the light phase. The incisions were closed with sutures.

\section{Measuring wheel-running activity}

Each mouse was housed in a separate cage $(183 \times 340 \times 148 \mathrm{~mm}$; CL-0135, CLEA Japan, Tokyo Japan $)$ with a running wheel $(12 \mathrm{~cm}$ diameter, SANKO, Osaka, Japan) 2 weeks after the surgery. The cages were placed in light-tight, ventilated boxes in which the light intensity at the bottom of the cage was 200-300 lx. The number of wheel revolutions was counted by a magnet-sensor-activated signal between a button magnet on the running wheel and a magnet relay (59070-010, Littelfuse, Inc., Chicago, IL, USA) fixed on a side wall of the cage, and was fed into a computer every minute. A chronobiology kit (Stanford Software Systems, Naalehu, HI, USA) and the ClockLab software (version 2.72, Actimetrics, Wilmette, IL, USA) were used to collect and display the activity data. Periods of wheel-running activity for 15 days in the DD condition were calculated with chisquared periodograms. Daily activities for 7 days in a normal light/dark (LD) cycle and 15 days in DD were quantified using the activity profile function in ClockLab.

\section{Gonadal gland weights and serum testosterone concentrations}

Animals had their LD cycles re-entrained for at least 2 weeks following wheel-running recordings in the DD condition. They were killed at $2-4 \mathrm{~h}$ before lights off under isoflurane anesthesia. Seminal vesicles, prostates, testes, and blood were collected. The body, seminal vesicle, prostate, and testis weights were measured. The blood samples were allowed to clot by leaving them undisturbed at room temperature $\left(22-25^{\circ} \mathrm{C}\right)$ for $30 \mathrm{~min}$. The clots were removed by centrifuging at $1000-2000 \times g$ for $10 \mathrm{~min}$ at $4{ }^{\circ} \mathrm{C}$. The sera were stored at $-80{ }^{\circ} \mathrm{C}$ until use.

Serum testosterone concentrations were measured in duplicate using an enzyme-linked immunosorbent assay kit (Testosterone Parameter Assay Kit, R\&D systems, Minneapolis, MN) according to the manufacturer's instructions.

\section{Experimental scheme}

\section{Experiment 1: effects of TP on the circadian rhythms of wheel-running activity in old mice}

Male C57BL6/J mice, 18 to 22 months old, were used as "old" mice in this experiment [31]. We divided intact old male mice into TP $(n=7)$ and vehicle $(n=9)$ groups. Animals in both groups were subcutaneously implanted with silicone tubes filled with TP or vehicle, respectively. After 
2 weeks of recovery, wheel-running activity was recorded in the DD condition for 15 days following 7 days of an LD cycle. Animals had their LD cycles re-entrained for at least 2 weeks following wheel-running recordings in the DD condition. They were killed $2-4 \mathrm{~h}$ before lights off under isoflurane anesthesia. Seminal vesicles, testes, and blood were collected.

\section{Experiment 2: effects of TP on the circadian rhythms of wheel-running activity in young mice}

Male C57BL6/J mice, 3 to 5 months old, were used as "young" mice in this experiment [27]. Young mice underwent GDX and were subcutaneously implanted with silicone tubes filled with TP (GDX + TP group, $n=6)$ or vehicle (GDX + vehicle group, $n=9$ ), respectively. After 2 weeks of recovery, wheel-running activity was recorded in the DD condition for 15 days following 7 days of an LD cycle. Animals had their LD cycles re-entrained for at least 2 weeks following wheel-running recordings in the DD condition. They were killed 2-4 h before lights off under isoflurane anesthesia. Seminal vesicles, prostates, and blood were collected.

\section{Experiment 3: effects of TP on the circadian rhythms of PER2::LUC mice in SCN explants}

We recorded bioluminescence rhythms in SCN explants from intact young (3- to 5-month-old) male PER2::LUC mice. We administered $10 \mu \mathrm{M}$ TP diluted with dimethyl sulfoxide (DMSO) (TP group, $n=7$ ) or vehicle (vehicle group, $n=7)$. Culture procedures were performed as described previously [32]. Briefly, 1-2 h before lights off, mice were euthanized by isoflurane anesthesia and rapidly decapitated. To prepare SCN cultures, brain tissue was removed and placed in chilled Hanks' buffered salt solution. The brain was sliced in the coronal plane on a microslicer (Dosaka EM, Kyoto, Japan) at a thickness of $300 \mu \mathrm{m}$. The bilateral SCN and minimal surrounding tissue were isolated from the slice using scalpels. SCN sections were placed at the liquid interface on membranes (Millicell-CM, PICM030-50; Merck Millipore, Burlington, MA, USA) in 35-mm dishes (Thermo Fisher Scientific, Waltham, MA, USA) containing $1.0 \mathrm{ml}$ of recording medium [serum-free, no sodium bicarbonate, no phenol red, Dulbecco's modified Eagle's medium (D-2902; SigmaAldrich) supplemented with $0.35 \mathrm{~g} / \mathrm{l}$ sodium bicarbonate, 10 mM HEPES (pH 7.2), B27 (2\%, 17504-010; Thermo Fisher Scientific)], $0.1 \mathrm{mM}$ luciferin (beetle luciferin, potassium salt; Wako Chemicals, Tokyo, Japan), and antibiotics (25 U/ml penicillin, $25 \mathrm{mg} / \mathrm{ml}$ streptomycin; Thermo Fisher Scientific).

TP was dissolved in DMSO (Sigma-Aldrich) and added directly to the recording medium. For controls, cultures were treated with DMSO vehicle. The concentration of DMSO did not exceed $1.0 \mu \mathrm{l} / \mathrm{ml}$ in the media. Following the addition of steroid or vehicle, the dishes were sealed with a cover using vacuum grease and placed under photomultiplier tubes (H6240; Hamamatsu, Hamamatsu, Japan) inside light-tight $35.0{ }^{\circ} \mathrm{C}$ environmental chambers. Bioluminescence was counted every minute from every dish for 6 days.

The data were detrended by subtraction of the 24-h running average from the raw data and then smoothed with a 2-h running average. The first $12 \mathrm{~h}$ of recording were not used in the analyses. The peak phase was set at the highest point of the smoothed data in each cycle. The period was calculated by measuring the mean interval of the peaks for four cycles. The amplitude was determined using the highest and lowest points in the second cycle.

\section{Statistics}

All results are presented as mean \pm standard error of the mean and were considered significant at $p<0.05$. Student's $t$-tests were used to examine differences between two groups.

\section{Results}

\section{Experiment 1: effects of TP on the circadian rhythms of wheel-running activity in old mice}

Both the TP and vehicle groups showed diurnal and circadian wheel-running rhythms in both the LD and DD conditions (Fig. 1a). We calculated the free-running period and daily wheel-running revolutions. No significant differences in the free-running period were observed between the TP and vehicle groups in the DD condition (Fig. 1b, TP: $23.85 \pm 0.04 \mathrm{~h}$; vehicle: $23.71 \pm 0.08 \mathrm{~h}$ ). Conversely, TP treatment significantly decreased daily wheel-running revolutions in both the LD (Fig. 1c, TP: 10,238 \pm 2031 revolutions/day; vehicle: $25,010 \pm 2713$ revolutions/day; $p<0.001$, Student's $t$-test) and DD (Fig. 1d, TP: 10,873 \pm 2084 revolutions/cycle; vehicle: $22,698 \pm 3219$ revolutions/cycle; $p<0.05$, Student's $t$-test) conditions.

To assess whether TP treatment affected other physiological parameters, we measured serum testosterone and the weights of the seminal vesicles and testis after behavioral recordings. The effects we observed agreed with the known effects of TP treatment, as we observed significant increases in the weight of seminal vesicles (Fig. 1e, TP: $63.89 \pm 7.38 \mathrm{mg} / \mathrm{g}$ body weight; vehicle: $41.78 \pm 5.33 \mathrm{mg} / \mathrm{g}$ body weight; $p<0.05$, Student's $t$-test) and serum concentration of testosterone (Fig. $1 \mathrm{~g}$, TP: $11.21 \pm 1.15 \mathrm{ng} / \mathrm{ml}$; vehicle: $3.15 \pm 1.25 \mathrm{ng} / \mathrm{ml} ; p<0.01$, Student's $t$-test), and decreases 

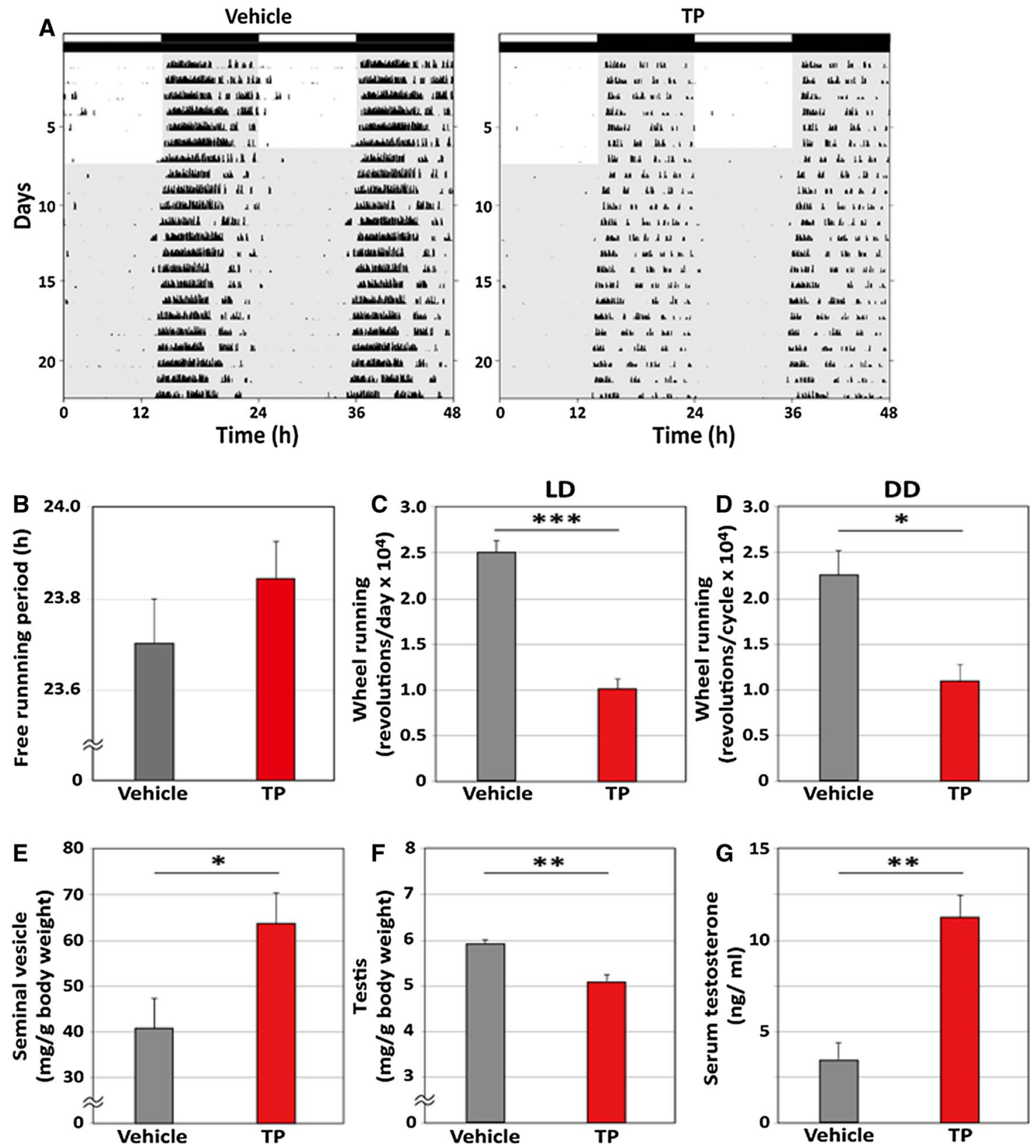

Fig. 1 Effects of testosterone propionate on the circadian rhythms of wheel-running activity in old mice. a Representative double-plotted actograms showing the wheel-running activity of old intact mice implanted with vehicle or testosterone propionate (TP) under light/ dark (LD) and constant darkness (DD) conditions. The light schedule is indicated by black and white bars above the record. b The freerunning period of wheel running activity in DD is shown. The daily

in the weights of the testis (Fig. 1f, TP: $5.09 \pm 0.25 \mathrm{mg} / \mathrm{g}$ body weight; vehicle: $5.90 \pm 0.14 \mathrm{mg} / \mathrm{g}$ body weight; $p<0.01$, Student's $t$-test). amounts of wheel running activity are shown in $\mathbf{c}$ LD and $\mathbf{b}$ DD. e, $\mathbf{f}$ The weights of the seminal vesicles and testis are shown. The weights were calculated as ratios of body weights. $\mathbf{g}$ The concentrations of serum testosterone are shown. Data are presented as mean \pm standard error of the mean, $n=7-9$ per group, $* p<0.05$, $* * p<0.01$, $* * * p<0.001$ for vehicle vs TP (Student's $t$-test)

\section{Experiment 2: effects of TP on the circadian rhythms of wheel-running activity in young mice}

We next investigated the effects of TP on circadian rhythms of wheel-running activity in young mice. Both the GDX + TP and GDX + vehicle groups showed diurnal 
and circadian wheel-running rhythms in both the LD and DD conditions (Fig. 2a). We calculated the free-running period and daily wheel-running revolutions. No significant differences in the free-running period were observed between the GDX + TP and GDX + vehicle groups in the DD condition (Fig. 2b, GDX + TP: $23.84 \pm 0.11 \mathrm{~h}$; GDX + vehicle: $23.77 \pm 0.03 \mathrm{~h}$ ). Conversely, TP treatment significantly increased daily wheel-running revolutions in both the LD (Fig. 2c, GDX + TP: 17,998 \pm 3413 revolutions/ day; GDX + vehicle: $3756 \pm 704$ revolutions/day; $p<0.001$,
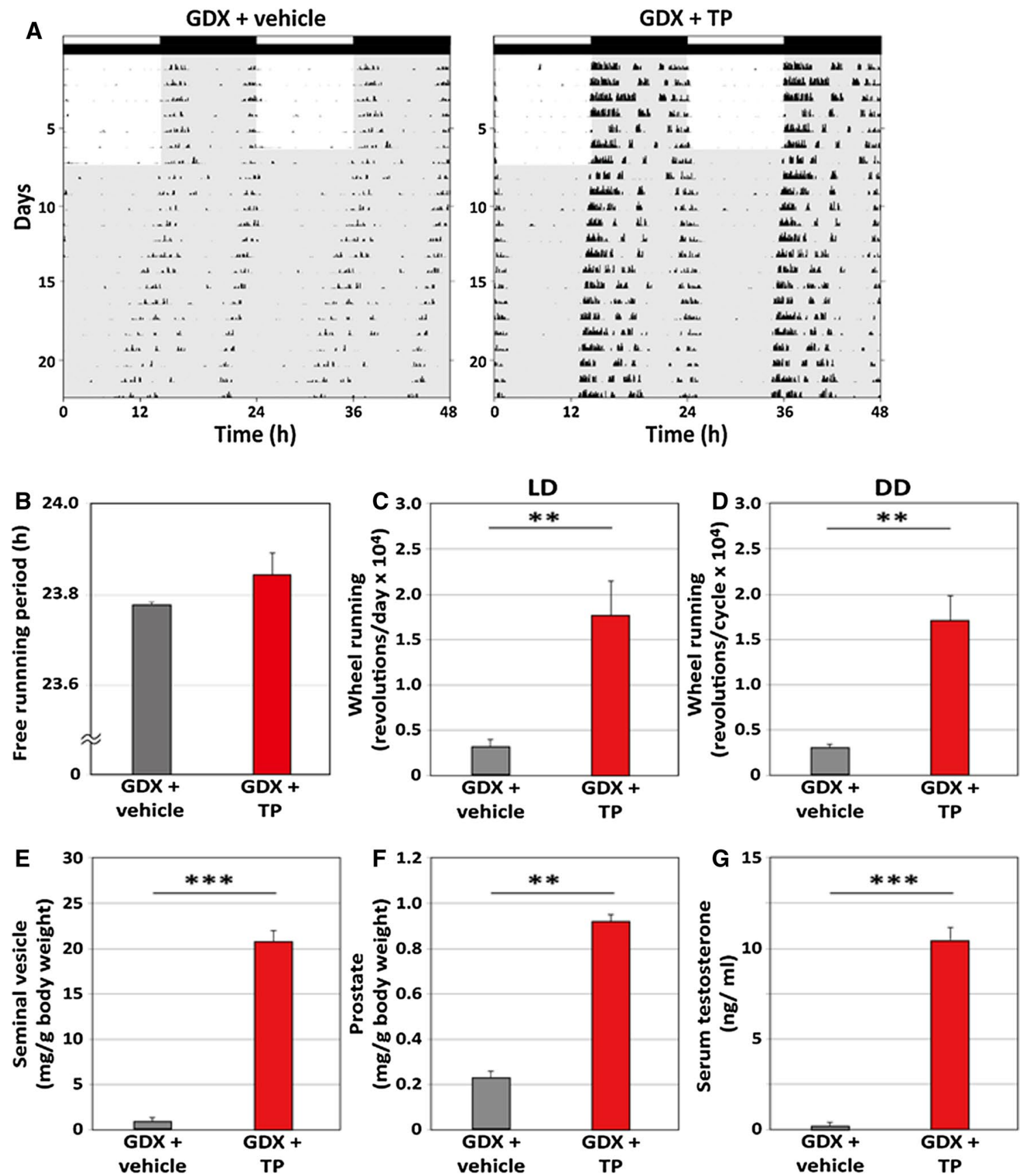

Fig. 2 Effects of testosterone propionate on the circadian rhythms of wheel-running activity in young mice. a Representative doubleplotted actograms showing the wheel-running activity of young GDX mice implanted with vehicle or testosterone propionate (TP) under light/dark (LD) and constant darkness (DD) conditions. The light schedule is indicated by black and white bars above the record. $\mathbf{b}$ The free-running period of wheel running activity in DD is shown. c, d
The daily amounts of wheel running activity are shown in $\mathbf{c}$ LD and d DD. e, f The weights of seminal vesicles and prostates are shown. The weights were calculated as ratios of body weights. $\mathrm{g}$ The concentrations of serum testosterone are shown. Data are presented as mean \pm standard error of the mean, $n=6-9$ per group, ${ }^{*} p<0.05$, $* * p<0.01, * * * p<0.001$ for vehicle vs TP (Student's $t$-test) 
Student's $t$-test) and DD (Fig. 2d, GDX + TP: 16,916 \pm 3063 revolutions/cycle; GDX + vehicle: $3137 \pm 326$ revolutions/ cycle; $p<0.001$, Student's $t$-test) conditions.

The effects we observed corroborated the known effects of TP treatment, as significant increases in the weights of the seminal vesicles (Fig. 2e, GDX + TP: $20.57 \pm 0.66 \mathrm{mg} / \mathrm{g}$ body weight; GDX + vehicle, $0.87 \pm 0.20 \mathrm{mg} / \mathrm{g}$ body weight; $p<0.001$, Student's $t$-test), prostates (Fig. 2f, GDX + TP: $0.93 \pm 0.22 \mathrm{mg} / \mathrm{g}$ body weight; GDX + vehicle: $0.23 \pm 0.03 \mathrm{mg} / \mathrm{g}$ body weight; $p<0.01$, Student's $t$-test), and serum concentrations of testosterone (Fig. $2 \mathrm{~g}, \mathrm{GDX}+\mathrm{TP}$ : $10.59 \pm 0.12 \mathrm{ng} / \mathrm{ml} ; \mathrm{GDX}+$ vehicle: $0.27 \pm 0.12 \mathrm{ng} / \mathrm{ml}$; $p<0.001$, Student's $t$-test) were observed.

\section{Experiment 3: effects of TP on the circadian rhythms of PER2::LUC in SCN explants}

We recorded bioluminescence rhythms in SCN explants from young male intact PER2::LUC mice. We applied $10 \mu \mathrm{M}$ TP diluted with DMSO (TP group, $n=7$ ) or vehicle (vehicle group, $n=7$ ). Both TP and vehicle-treated SCN tissues clearly showed circadian rhythms in PER2::LUC expression (Fig. 3a). We calculated the period and amplitude of PER2::LUC expression. TP treatment significantly lengthened the circadian period in the SCN (Fig. 3b, TP: $25.69 \pm 0.19 \mathrm{~h}$; vehicle: $25.01 \pm 0.17 \mathrm{~h} ; p<0.05$, Student's $t$-test), while TP did not affect the amplitude of the PER2::LUC rhythm (Fig. 3c, TP: $7025 \pm 572.9$ counts/min; vehicle: $5589 \pm 1277.0$ counts $/ \mathrm{min})$.

\section{Discussion}

Although our results demonstrate that TP influences the circadian rhythm of wheel-running activity and PER2::LUC rhythm in the SCN, they are inconsistent with our hypothesis. TP treatment increased the daily wheel-running activity in young mice, contrary to the effects in old mice. Additionally, TP treatment lengthened the periods of PER2::LUC rhythm in SCN explants from young mice without affecting the amplitude of the same. In a previous result, with regards to in vivo multiunit neural activity recordings from the SCN of freely moving young and old mice, we observed that the amplitude of diurnal and circadian rhythms of neural activity was reduced in older mice. The reduction simultaneously occurred with a reduction in the wheel running activity rhythm [33]. These results indicate that aging associated degradation of the circadian neural output leads to an age-related decline in circadian behavioral rhythm. It is our understanding that TP treatment reduces the wheel running activity by further exacerbating the amplitude of neural firing activity rhythm in the SCN. Thus, our data suggests
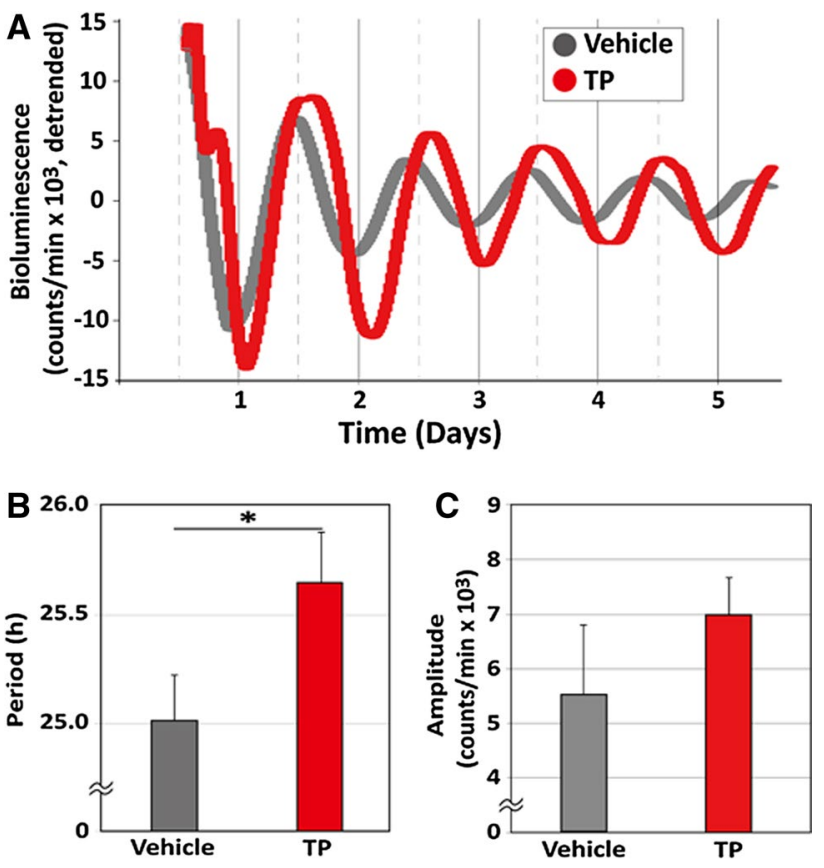

Fig. 3 Effects of testosterone propionate on the circadian rhythms of PER2::LUC in suprachiasmatic nucleus explants. a Representative bioluminescence traces showing the effects of testosterone propionate (TP) on circadian rhythms of PERIOD2::LUCIFERASE (PER2::LUC) expression in cultured suprachiasmatic nucleus (SCN) explants from young male mice. The SCN was treated with vehicle [dimethylsulfoxide (DMSO)] or $10 \mu \mathrm{M}$ TP. The $\mathbf{b}$ mean period and c amplitude values are shown for each group. Data are presented as mean \pm standard error of the mean, $n=7$ per group, ${ }^{*} p<0.05$, for vehicle vs TP (Student's $t$-test)

that TP treatment does not improve age-related decline in circadian rhythms.

In old mice, we confirmed the known effects of TP treatment, as we observed significant increases in the weights of the seminal vesicles and testosterone levels in the circulating blood and decreased testis weights [34]. However, TP treatment did not restore age-related changes, such as lengthening of the circadian period and decreases in circadian amplitude. As it is known that testosterone influences the autonomic system [35] and has anabolic effects on skeletal muscle mass and bone architecture in humans and mice [36-39], the result of decreasing activity levels was very surprising.

TRT has become popular as a treatment to alleviate agerelated decline in physical and mental function in elderly men [5]. However, TRT did not increase the walking distance in a 6-min walk test in a physical function trial [3]. In addition, it has been reported that TRT produces a number of adverse effects including worsening of sleep apnea in older men [40]. Collectively, TRT does not seem to improve all age-related declines in physical function in humans. In this regard, the present results are consistent with the data from 
human clinical studies [3]. The present results in old mice suggest that testosterone treatment does not improve circadian behavioral rhythms in old mice.

In the present study, TP treatment increased the activity levels in young GDX mice. Daan et al. [26] reported on the effects of androgens on circadian behavioral rhythms and indicated that GDX lengthened the free-running period and reduced wheel-running activity in mice. Both effects were abolished by implantation of TP. These findings suggest that TP treatment shortens the free-running period and increases activity levels [26]. In the present study, however, TP treatment administered to young GDX mice affected the activity levels but did not shorten the free-running periods of wheelrunning rhythm. The discrepancy may be explained by the findings of a recent paper [27], which revealed that GDX lengthened the free-running period in dim light conditions, but not in complete DD conditions, and increasing intensities of constant light parametrically increased the free-running period. The present behavioral study was performed in complete DD conditions. Therefore, the absence of an effect of TP treatment in young GDX mice on the free-running period may be due to differences in the light conditions. However, a recent study of high-throughput chemical screening of existing drugs for circadian clock modulators showed that dehydroepiandrosterone, one of the most abundant circulating androgens in humans, shortened free-running periods of wheel-running activity rhythms in young male mice [41]. Thus, androgens may have the potential to alter the period of circadian behavioral rhythms.

In SCN explants, we observed that $10 \mu \mathrm{M}$ TP treatment significantly lengthened the period of PER2::LUC expression but did not affect its amplitude. Although Silver's group reported that a direct $\mathrm{TP}$ implant to the SCN altered the period of circadian behavioral rhythms [25], the present study is the first to show a direct effect of testosterone on the circadian rhythms of clock genes in the SCN. Regarding the changing rhythms of clock gene expression by testosterone in peripheral tissues, it has been reported that GDX attenuates the expression rhythm of Per 2 mRNA, and testosterone administration partially rescues the expression levels of Per2 [42]. Using the Per2-dluc reporting system, it was demonstrated that $100 \mathrm{nM}$ testosterone treatment increased the amplitude of the Per2-dluc rhythm in mesenchyme cells but did not affect the period [42], suggesting that testosterone directly influences circadian rhythms of clock gene expression in peripheral tissues. It is known that androgen receptors are expressed in the SCN of humans and rodents, and that the SCN is sensitive to androgen treatment [21-25]. Thus, it is likely that testosterone alters the circadian rhythms of cellular clocks in the SCN.

In conclusion, TP alters circadian rhythms of PER2::LUC in the SCN and increases locomotor activity levels in young male mice; however, the same treatment worsens the amplitude of circadian wheel running rhythm in aged male mice. Testosterone replacement therefore seems to exacerbate the age-related decline in circadian rhythmicity. These findings indicate the need for careful dissection of the utility of TRT for age-related sleep disorders.

Author contributions WN and TJN designed the study; SF, AH, and TJN performed the experiments; $\mathrm{SF}, \mathrm{AH}$, and TJN analyzed the data; $\mathrm{WN}$ and TJN wrote the paper.

Funding This work was supported by JSPS KAKENHI Grant numbers $17 \mathrm{H} 04022$.

\section{Compliance with ethical standards}

Conflict of interest The authors have nothing to disclose.

Ethical approval All applicable international, national, and/or institutional guidelines for the care and use of animals were followed. The protocol was approved by the Institutional Animal Care and Use Committee at the School of Agriculture, Meiji University.

\section{References}

1. Harman SM, Metter EJ, Tobin JD, Pearson J, Blackman MR (2001) Longitudinal effects of aging on serum total and free testosterone levels in healthy men. Baltimore Longitudinal Study of Aging. J Clin Endocrinol Metab 86:724-731. https://doi. org/10.1210/jcem.86.2.7219

2. Machida T, Yonezawa Y, Noumura T (1981) Age-associated changes in plasma testosterone levels in male mice and their relation to social dominance or subordinance. Horm Behav $15: 238-245$

3. Snyder PJ, Ellenberg SS, Farrar JT (2016) Testosterone treatment in older men. N Engl J Med 375:90. https://doi.org/10.1056/ NEJMc1603665

4. Shores MM, Moceri VM, Gruenewald DA, Brodkin KI, Matsumoto AM, Kivlahan DR (2004) Low testosterone is associated with decreased function and increased mortality risk: a preliminary study of men in a geriatric rehabilitation unit. J Am Geriatr Soc 52:2077-2081. https://doi.org/10.111 1/j.1532-5415.2004.52562.x

5. Tsametis CP, Isidori AM (2018) Testosterone replacement therapy: for whom, when and how? Metabolism 86:69-78. https://doi. org/10.1016/j.metabol.2018.03.007

6. Spitzer M, Huang G, Basaria S, Travison TG, Bhasin S, (2013) Risks and benefits of testosterone therapy in older men. Nat Rev Endocrinol 9:414-424. https://www.nature.com/articles/nrend o.2013.73

7. Bliwise DL (1993) Sleep in normal aging and dementia. Sleep 16:40-81

8. Turek FW, Penev P, Zhang Y, van Reeth O, Zee P (1995) Effects of age on the circadian system. Neurosci Biobehav Rev 19:53-58

9. Van Someren EJ (2000) Circadian rhythms and sleep in human aging. Chronobiol Int 17:233-243

10. Descovich GC, Montalbetti N, Kühl JF, Rimondi S, Halberg F, Ceredi C (1974) Age and catecholamine rhythms. Chronobiologia $1: 163-171$ 
11. Gubin D, Cornélissen G, Halberg F, Gubin G, Uezono K, Kawasaki T (1997) The human blood pressure chronome: a biological gauge of aging. In Vivo 11:485-494

12. Nelson W, Bingham C, Haus E, Lakatua DJ, Kawasaki T, Halberg F (1980) Rhythm-adjusted age effects in a concomitant study of twelve hormones in blood plasma of women. J Gerontol 35:512-519

13. Pittendrigh CS, Daan S (1974) Circadian oscillations in rodents: a systematic increase of their frequency with age. Science 186:548-550

14. Scarbrough K, Losee-Olson S, Wallen EP, Turek FW (1997) Aging and photoperiod affect entrainment and quantitative aspects of locomotor behavior in Syrian hamsters. Am J Physiol 272:R1219R1225. https://doi.org/10.1152/ajpregu.1997.272.4.R1219

15. Valentinuzzi VS, Scarbrough K, Takahashi JS, Turek FW (1997) Effects of aging on the circadian rhythm of wheel-running activity in C57BL/6 mice. Am J Physiol 273:R1957-R1964

16. Zhang Y, Kornhauser JM, Zee PC, Mayo KE, Takahashi JS, Turek FW (1996) Effects of aging on light-induced phase-shifting of circadian behavioral rhythms, fos expression and CREB phosphorylation in the hamster suprachiasmatic nucleus. Neuroscience 70:951-961

17. Nakamura TJ, Takasu NN, Nakamura W (2016) The suprachiasmatic nucleus: age-related decline in biological rhythms. J Physiol Sci 66:367-374. https://doi.org/10.1007/s12576-016-0439-2

18. Meyer-Bernstein EL, Jetton AE, Matsumoto SI, Markuns JF, Lehman MN, Bittman EL (1999) Effects of suprachiasmatic transplants on circadian rhythms of neuroendocrine function in golden hamsters. Endocrinology 140:207-218. https://doi.org/10.1210/ endo.140.1.6428

19. Partch CL, Green CB, Takahashi JS (2014) Molecular architecture of the mammalian circadian clock. Trends Cell Biol 24:90-99

20. Yoo SH, Yamazaki S, Lowrey PL, Shimomura K, Ko CH, Buhr ED, Siepka SM, Hong HK, Oh WJ, Yoo OJ, Menaker M, Takahashi JS (2004) PERIOD2:LUCIFERASE real-time reporting of circadian dynamics reveals persistent circadian oscillations in mouse peripheral tissues. Proc Natl Acad Sci USA 101:53395346. https://doi.org/10.1073/pnas.0308709101

21. Zhou L, Blaustein JD, De Vries GJ (1994) Distribution of androgen receptor immunoreactivity in vasopressin- and oxytocinimmunoreactive neurons in the male rat brain. Endocrinology 134:2622-2627. https://doi.org/10.1210/endo.134.6.8194487

22. Fernández-Guasti A, Kruijver FP, Fodor M, Swaab DF (2000) Sex differences in the distribution of androgen receptors in the human hypothalamus. J Comp Neurol 425:422-435

23. Karatsoreos IN, Wang A, Sasanian J, Silver R (2007) A role for androgens in regulating circadian behavior and the suprachiasmatic nucleus. Endocrinology 148:5487-5495. https://doi. org/10.1210/en.2007-0775

24. Karatsoreos IN, Butler MP, Lesauter J, Silver R (2011) Androgens modulate structure and function of the suprachiasmatic nucleus brain clock. Endocrinology 152:1970-1978. https://doi. org/10.1210/en.2010-1398

25. Model Z, Butler MP, LeSauter J, Silver R (2015) Suprachiasmatic nucleus as the site of androgen action on circadian rhythms. Horm Behav 73:1-7. https://doi.org/10.1016/j.yhbeh.2015.05.007

26. Daan S, Damassa D, Pittendrigh CS, Smith ER (1975) An effect of castration and testosterone replacement on a circadian pacemaker in mice (Mus musculus). Proc Natl Acad Sci USA 72:3744-3747

27. Butler MP, Karatsoreos IN, LeSauter J, Silver R (2012) Dosedependent effects of androgens on the circadian timing system and its response to light. Endocrinology 153:2344-2352. https:// doi.org/10.1210/en.2011-1842

28. Dziuk PJ, Cook B (1966) Passage of steroids through silicone rubber. Endocrinology 78:208-211

29. Kincl FA, Benagiano G, Angee I (1968) Sustained release hormonal preparations. 1. Diffusion of various steroids through polymer membranes. Steroids 11:673-680

30. Kratochvíl P, Benagiano G, Kincl FA (1970) Sustained release hormonal preparations. 6. Permeability constant of various steroids. Steroids 15:505-511

31. Nakamura TJ, Nakamura W, Tokuda IT, Ishikawa T, Kudo T, Colwell CS, Block GD (2015) Age-related changes in the circadian system unmasked by constant conditions. eNeuro. https://doi. org/10.1523/ENEURO.0064-15.2015

32. Nakamura TJ, Sellix MT, Menaker M, Block GD (2008) Estrogen directly modulates circadian rhythms of PER2 expression in the uterus. Am J Physiol Endocrinol Metab 295:E1025-E1031. https ://doi.org/10.1152/ajpendo.90392.2008

33. Nakamura TJ, Sellix MT, Menaker M, Block GD (2011) Agerelated decline in circadian output. J Neurosci 31:10201-10205

34. Vanderschueren D, Vandenput L, Boonen S, Van Herck E, Swinnen JV, Bouillon R (2000) An aged rat model of partial androgen deficiency: prevention of both loss of bone and lean body mass by low-dose androgen replacement. Endocrinology 141:1642-1647. https://doi.org/10.1210/endo.141.5.7472

35. Keast JR (2000) Effects of testosterone on pelvic autonomic pathways: progress and pitfalls. J Auton Nerv Syst 79:67-73

36. Bhasin S, Storer TW, Berman N, Callegari C, Clevenger B, Phillips J, Bunnell TJ, Tricker R, Shirazi A, Casaburi R (1996) The effects of supraphysiologic doses of testosterone on muscle size and strength in normal men. N Engl J Med 335:1-7

37. Köhn FM (2006) Testosterone and body functions. Aging Male 9:183-188

38. Matsumoto C, Inada M, Toda K, Miyaura C (2006) Estrogen and androgen play distinct roles in bone turnover in male mice before and after reaching sexual maturity. Bone 38:220-226

39. MacLean HE, Chiu WS, Notini AJ, Axell AM, Davey RA, McManus JF, Ma C, Plant DR, Lynch GS, Zajac JD (2008) Impaired skeletal muscle development and function in male, but not female, genomic androgen receptor knockout mice. FASEB J 22:2676-2689. https://doi.org/10.1096/fj.08-105726

40. Matsumoto AM (2002) Andropause: clinical implications of the decline in serum testosterone levels with aging in men. J Gerontol A Biol Sci Med Sci 57:M76-M99. https://doi.org/10.1016/j. tcb.2013.07.002

41. Tamai TK, Nakane Y, Ota W, Kobayashi A, Ishiguro M, Kadofusa N, Ikegami K, Yagita K, Shigeyoshi Y, Sudo M, NishiwakiOhkawa T, Sato A, Yoshimura T (2018) Identification of circadian clock modulators from existing drugs. EMBO Mol Med 10:e8724. https://doi.org/10.15252/emmm.201708724

42. Kawamura M, Tasaki H, Misawa I, Chu G, Yamauchi N, Hattori MA (2014) Contribution of testosterone to the clock system in rat prostate mesenchyme cells. Andrology 2:225-233. https://doi.org /10.1111/j.2047-2927.2013.00161.x

Publisher's Note Springer Nature remains neutral with regard to jurisdictional claims in published maps and institutional affiliations. 